\title{
Knowledge and attitudes of the students of the Niamey Faculty of Health Sciences on tobacco
}

\section{Connaissances et attitudes des étudiants de la Faculté des Sciences de la Santé de Niamey sur le tabagisme}

\author{
MM. Assao Neino', I. Alkassoum Salifou ${ }^{2}$, MA. Gagara Issoufou' ${ }^{1}$ A. Haj Daouda ${ }^{3}$, \\ AR. Ouédraogo ${ }^{4}$, AR. Mahaman Mallam ${ }^{1}$ \\ 1: Service de Pneumo-Phtisiologie de l'Hôpital National Lamordé - HNL \\ 2: Direction de la Surveillance de la Riposte aux Épidémies \\ 3: Faculté des Lettres et Sciences Humaines \\ 4: Centre Hospitalier Universitaire Yalgado-Ouedraogo. Burkina Faso
}

\begin{abstract}
During these last 10 years we note a growing consumption of the tobacco especially that of chicha by the young Nigeriens. This pushed us to do this work. It was a prospective cross-sectional study over 20 months (January 1, 2016 to August 31 , 2017) for 601 students (1st to 7th year Medicine and 1st to 3rd year Pharmacy) of the Faculty of Health Sciences (FSS) of Niamey. The data had been collected on a survey card after an interview. The smoker was the student declared daily or casual smoker at the time of the survey. A total of 601 students from the Niamey FSS had been collected. The prevalence of smoking was $10.64 \%$. Males accounted for $53.40 \%$ and the average age was 22.55 years. Shisha was the most common and smoked form of smoking by women at $76.48 \%$. The average daily cost of tobacco was 150 to 300 FCFA for $66.66 \%$ of smokers. According to the Fagerström test, $10 \%$ of daily smokers were highly addicted. For $33.50 \%$ of students smoking shisha was not considered smoking, $55.30 \%$ did not know that shisha is more harmful than smoking. Daily smokers who tried to quit accounted for 95\% (19 cases). Other smoking habits (alcohol and / or cannabis) added to smoking in $65.70 \%$ of current smokers. The prevalence of smoking among students in our study is low but relatively high for a population that will have the role of protecting society from the harmful effects of tobacco. However, special attention must also be given to the growing consumption of chicha in our society.
\end{abstract}

KEYWORDS: Knowledge; Attitudes; Smoking; Faculty of Health Sciences; Niamey.

\section{RÉSUMÉ}

Pendant ces 10 dernières années nous constatons une consommation grandissante du tabac notamment celle de la chicha par les jeunes Nigériens. Ceci nous a poussés à réaliser ce travail. C'était une étude prospective transversale sur 20 mois (1re Janvier 2016 au 31 Aout 2017) chez 601 étudiants (1re à 7me année Médecine et 1re à 3 me année Pharmacie) de la Faculté des Sciences de la Santé (FSS) de Niamey. Les données avaient été recueillies sur une fiche d'enquête après un entretien. Le fumeur était l'étudiant déclaré fumeur quotidien ou occasionnel au moment de l'enquête. Au total 601 étudiants de la FSS de Niamey avaient été colligés. La prévalence du tabagisme était de 10,64\%. Le sexe masculin représentait 53,40\% et l'âge moyen était de 22,55 ans. La chicha était le mode de consommation du tabac le plus fréquent et le plus fumé par les femmes avec 76,48\%. Le coût moyen journalier du tabac était de 150 à 300 FCFA pour 66,66\% des fumeurs. Selon le test de Fagerström, $10 \%$ des fumeurs quotidiens présentaient une forte dépendance. Pour 33,50\% des étudiants fumer de la chicha n'était pas considéré comme du tabagisme, 55,30\% ignoraient que la chicha est plus nocive que la cigarette. Les fumeurs quotidiens qui avaient essayé d'arrêter de fumer représentaient 95\% (19 cas). D'autres habitudes toxiques (alcool et ou cannabis) s'ajoutaient au tabagisme chez $65,70 \%$ des fumeurs actuels. La prévalence du tabagisme chez les étudiants dans notre étude est faible mais relativement élevée pour une population qui aura le rôle de protéger la société contre les méfaits du tabac. Cependant une attention particulière doit aussi être accordée à la consommation grandissante de la chicha dans notre société.

MOTS CLÉS: Connaissances; Attitudes; Tabagisme; Faculté des Sciences de la Santé, Niamey. 


\section{INTRODUCTION}

La lutte contre le tabagisme est devenue l'une des premières préoccupations de santé publique et l'un des principaux objectifs du plan de lutte contre le cancer [1]. Les pharmaciens et les médecins sont les professionnels de la santé de première ligne face aux millions de fumeurs candidats à l'arrêt [2]. Plusieurs études ont rapporté que les étudiants en médecine et pharmacie en fin d'étude étaient peu préparés à ce rôle et que leurs attitudes à l'égard des fumeurs dépendaient de leur comportement personnel en matière du tabac [3-5]. Le Niger dispose d'une loi antitabac mais il n'existe pas de loi et de réglementation contre la chicha. Pendant ces 10 dernières années au Niger nous constatons une augmentation de la consommation du tabac notamment celle de la chicha par les jeunes et il n'existe pas de module d'enseignement de la pathologie du tabac à la FSS. Ceci nous a poussés à réaliser ce travail.

\section{MÉTHODES}

C'était une étude prospective transversale sur 20 mois (1 re Janvier 2016 au 31 Aout 2017) chez 601 étudiants (1re à 7 me année Médecine et $1^{\text {re }}$ à 3 me année Pharmacie) de la FSS de Niamey. Un quota de $25 \%$ du nombre des étudiants inscrits au mois de Mars de l'année académique 2015-2016 dans chaque promotion a été enquêté. La Faculté des Sciences de la Santé avait été créé en 1984 et occupe ses propres bâtiments dans la partie occidentale du campus de l'Université Abdou Moumouni de Niamey. Elle est composée de 3 sections (Médecine, Pharmacie et chirurgie dentaire qui n'est pas encore fonctionnelle).

Les fumeurs actuels comprennent les fumeurs quotidiens qui sont des personnes qui fument un produit du tabac au moins une fois par jour, les fumeurs occasionnels sont ceux qui fument, mais pas tous les jours et l'ex-fumeur est celui qui a fumé au moins 100 cigarettes ou l'équivalent en tabac dans sa vie, mais qui ne fume pas du tout à present. Un questionnaire préétablie avait été élaborés sur la base des indicateurs de la Convention Cadre de la Lutte AntiTabac (CCLAT) de l'OMS 1ere édition conciliés au sous-ensemble de questions tirées de l'enquête mondiale sur le tabagisme chez les adultes (GATS : Global Adult Tobaco Survey) 2ème édition ; le tout adapté à notre contexte.

Les données avaient été recueillies sur une fiche d'enquête après un entretien. L'enquête s'est tenue dans l'anonymat, et la confidentialité. La participation à l'enquête de chaque sujet interrogé a été obtenue après un consentement éclairé de la part de l'enquêteur. Les données collectées avaient été traitées et analysées à l'aide du logiciel épi info version 3.5.1

\section{RÉSULTATS}

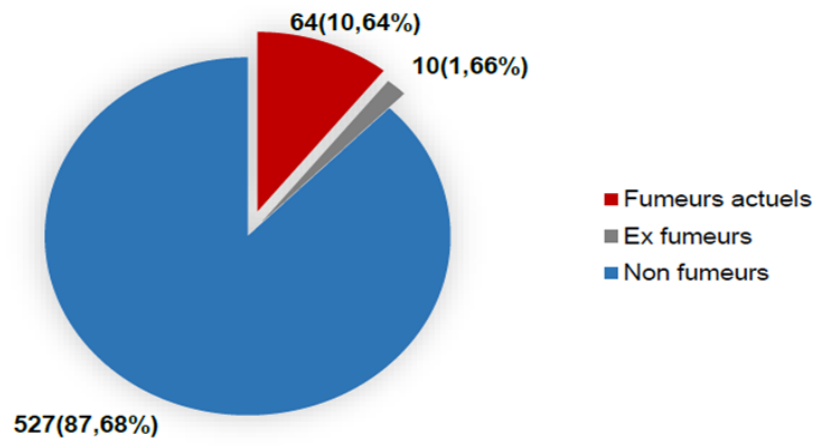

FIGURE 1. Répartition des étudiants en fonction de leur statut tabagique.

Au total 601 étudiants de la FSS avaient été colligés. Le sexe masculin représentait $53,40 \%$ et le Sex ratio (H/F) 1,14. L'âge moyen était de 22,55 ans avec des extrêmes allant de 17 et 32 ans. Les étudiants de médecine étaient au nombre de 578 (96,2\%) et ceux de la pharmacie à $23(3,8 \%)$. La prévalence du tabagisme était de 10,64\% voir (Figure 1). Les fumeurs quotidiens représentaient $31,25 \%$ (20 cas) et les occasionnels $68,75 \%$ (44 cas). La chicha était le mode de consommation du tabac le plus fréquent et le plus fumé par les femmes avec 76,48\% voir (Figure 2). Le coût moyen journalier du tabac était de 150 à 300 FCFA pour $66,66 \%$ des fumeurs voir (Figure 3).

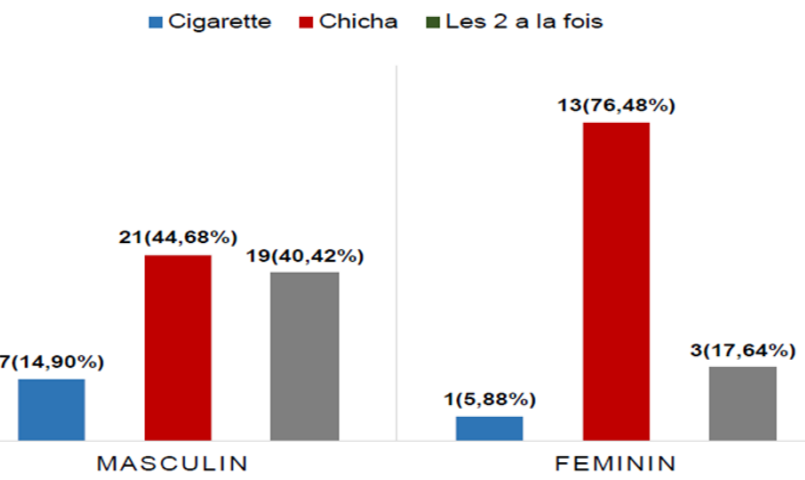

FIGURE 2. Répartitions des fumeurs selon le mode de consommation du tabac et le sexe.

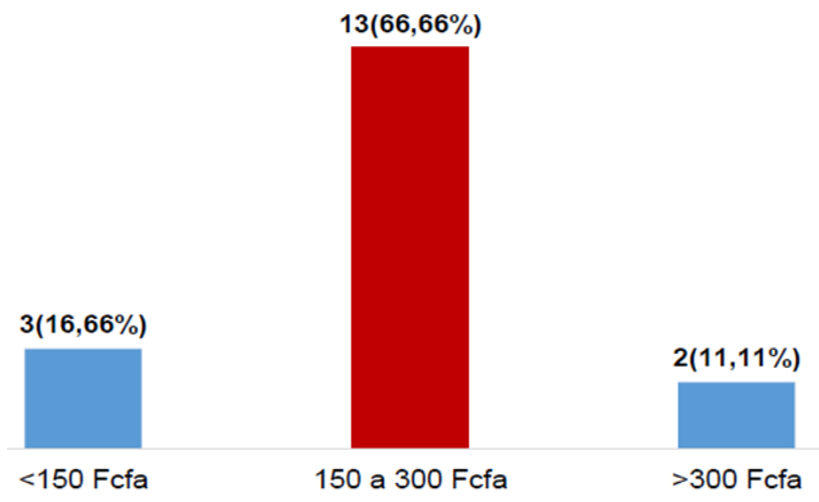

FIGURE 3. Répartition des fumeurs selon le cô̂t journalier du tabac. 
Selon le test de Fagerström, 10\% des fumeurs quotidiens présentaient une forte dépendance à la nicotine voir (Tableau 1).

\begin{tabular}{lcc} 
TABLEAU 1 & \multicolumn{2}{c}{$\begin{array}{l}\text { Répartition des fumeurs } \\
\text { diens selon le degré de dépendance } \\
\text { à la nicotine }\end{array}$} \\
\hline Dépendance à la nicotine & Fréquence & Pourcentage \\
\hline Faible & 6 & 30 \\
Moyenne & 12 & 60 \\
Forte & 2 & 10 \\
Total & $\mathbf{2 0}$ & $\mathbf{1 0 0}$ \\
\hline & &
\end{tabular}

Pour $33,50 \%$ des étudiants fumer de la chicha n'était pas considéré comme du tabagisme, 55,30\% ignoraient que la chicha est plus nocive que la cigarette et 90,30\% des étudiants pensaient qu'il fallait arrêter de fumer pour donner un bon exemple. L'existence d'une loi anti-tabac au Niger était connue par 23,43\% (15 cas) des étudiants fumeurs actuels, 30\% (3 cas) des ex-fumeurs et $18,40 \%$ (97 cas) des non-fumeurs. Les fumeurs quotidiens qui avaient essayé d'arrêter de fumer représentaient 95\% (19 cas). La mauvaise habitude, la nocivité du tabac et la religion étaient les principales raisons ayant motivées les fumeurs actuels à tenter d'arrêt le tabagisme voir (Tableau 2).

\section{TABLEAU 2 Répartition des fumeurs actuels en fonction des raisons des tentatives d'arrêt du tabagisme}

\begin{tabular}{lcc}
$\begin{array}{l}\text { Raisons des tentatives } \\
\text { d'arrêt du tabagisme }\end{array}$ & Fréquence & Pourcentage \\
\hline $\begin{array}{l}\text { La mauvaise habitude de } \\
\text { fumer le tabac }\end{array}$ & 11 & 17,2 \\
La nocivité du tabac & 10 & 15,6 \\
$\begin{array}{l}\text { Le respect de la religion } \\
\begin{array}{l}\text { Ne pas devenir dépendant } \\
\text { du tabac }\end{array}\end{array}$ & 10 & 15,6 \\
$\begin{array}{l}\text { Se sentir heureux } \\
\text { La peur de développer un } \\
\text { cancer }\end{array}$ & 8 & 12,5 \\
$\begin{array}{l}\text { Ne pas déranger les voisins } \\
\text { La pression de l'entourage } \\
\text { pour arrêter le tabac }\end{array}$ & 5 & 12,5 \\
$\begin{array}{l}\text { Le coût du tabac } \\
\text { Total }\end{array}$ & 5 & 7,8 \\
\hline & 2 & 7,8 \\
\hline
\end{tabular}

La religion et la mauvaise habitude étaient les principales raisons ayant motivées l'arrêt du tabagisme chez les ex fumeurs voir tableau III. D'autres habitudes toxiques (alcool et ou cannabis) s'ajoutaient au tabagisme chez $65,70 \%$ (42 cas) des fumeurs actuels. Ainsi on retrouvait alcool+ cannabis + tabagisme 22 cas $(34,40 \%)$, alcool + tabagisme 14 cas $(21,90 \%)$, cannabis + tabagisme 6 cas $(9,40 \%)$.

\section{DISCUSSION}

En 2011 en Afrique, l'OMS estimait que la prévalence $\mathrm{du}$ tabagisme des adultes (hommes et femmes) en Afrique subsaharienne se répartissait entre $5 \%$ au Niger et $34 \%$ au Sierra Leone. Les estimations de la prévalence du tabagisme en Afrique vont de $8 \%$ à 48 $\%$ chez les hommes adultes et de $0,4 \%$ à $20 \%$ chez les femmes adultes [6].

Au Niger le tabagisme affecte surtout les jeunes. Selon une enquête globale réalisée chez les jeunes en milieu scolaire par l'Association Nigérienne pour la Promotion de la Santé Publique (ANSP) en 2006 : $14,5 \%$ des élèves utilisent couramment un produit du tabac [7].

Dans notre étude la prévalence du tabagisme étude était de 10,64\%. SOLTANI MS avait retrouvé chez les étudiants en médecine une prevalance de $33 \%$ en Tinusie [8].

Ceci pourrait s'expliquer par nos différences culturelles car au Niger le tabagisme ne fait pas parti de notre culture contrairement aux autres pays du Maghreb qui ont une prévalence plus élevée.

Le narguilé et la cigarette étaient les 2 modes de consommation du tabac.

La chicha était le mode de consommation du tabac le plus fréquent et le plus fumé par les femmes avec $76,48 \%$ VS $44,68 \%$ chez les hommes. Cette utilisation prédominante du narguilé par les femmes pourrait s'expliquée par une méconnaissance des méfaits de la chicha, l'absence de loi et de réglementation sur cette dernière, et l'absence de module sur le tabac dans l'enseignement des étudiants à la FSS.

Le coût moyen journalier de la cigarette était de 150 à 300 FCFA par jour pour $66,66 \%$ des fumeurs quotidiens. Ce Coût est élevé pour un étudiant Nigérien qui à la chance d'avoir une bourse plaine qui est de 35.000FCFA par mois. Sur le plan économique le tabac affecte les familles à travers les dépenses en produits du tabac sans oublier le coût de la prise charge des maladies liées au tabagisme [9].

Selon le test de Fagerström, 10\% des fumeurs réguliers avaient une dépendance forte dans notre serie. THIAM N au Senegal en 2013 avait retrouvé une forte dépendance à la nicotine chez $35 \%$ des étudiants en Médecine [10]. La difficulté de sevrage croit 
avec le niveau de dépendance à la nicotine d'où l'importance d'une prévention primaire et la nécessité d'un programme d'aide au sevrage.

D'autres habitudes toxiques (alcool et ou cannabis) s'ajoutaient au tabagisme chez $65,70 \%$ des fumeurs actuels. Ainsi on retrouvait alcool+ cannabis + tabagisme $34,40 \%$, alcool + tabagisme $21,90 \%$, cannabis + tabagisme $9,40 \%$. Ce constat a été aussi fait par KOUARA S en 2013 qui avait retrouvé chez les fumeurs la prise du cannabis dans 71,80\%, l'alcool dans $66,70 \%$ [11]. Ainsi les fumeurs sont habituellement vulnérables à d'autres habitudes toxiques. En effet, la consommation d'alcool et la consommation de tabac sont liées car les étudiants qui consomment quotidiennement de l'alcool ont des probabilités plus élevés d'être des gros fumeurs.

La majorité des étudiants méconnaissaient l'existence d'une loi antitabac au Niger. Seulement 23,43\% des fumeurs actuels, 30\% des ex-fumeurs et $18,40 \%$

\section{CONFLIT D'INTÉRÊTS}

Aucun.

\section{RÉFÉRENCES}

1. DAUTZENBERG B. Traiter le tabagisme: une priorité en cancérologie pulmonaire. Rev Pneumol Clin 2004 ; $60: 308-311$

2. MAITRE B, HIRSCH A. Prise en charge de l'arrêt du tabac. Fiche technique 2007, p1-8.

3. TESSIER JF, FREOUR P, CROFTON J: Les etudiants en médecine français et le tabac. Rev Mal Respir 1988 ; 589-92.

4. TESSIER JF, FREOUR P : Smoking habit and attitudes of medical students toward smoking and antismoking compaigns in 9 asian countries. Int J Epidemiol 1992 ; 298-304.

5. RODNEY MC, FREOUR P : Smoking among medical students. Am J Public Health 1980 ; 70 :169-71.

6. Network of Africain Science Academies. Prévention d'une épidémie de tabagisme en Afrique : Un appel pour une action efficace visant à appuyer le développement sanitaire, social et économique, rapport de la commission sur les effets néfastes du tabac sur la santé, l'économie et le développement, 2014, p15-20. des non-fumeurs connaissaient l'existence de cette loi. Une étude au Maroc avait retrouvé 35,8\% d'étudiants qui connaissaient l'existence d'une loi antitabac en 2007 [12]. Ce pourcentage faible des étudiants qui connaissaient 'existence de la loi Nigérienne antitabac laisse prédire qu'il faut encore fournir beaucoup d'efforts dans le cadre de l'information.

\section{CONCLUSION}

La prévalence du tabagisme chez les étudiants dans notre étude est faible mais relativement élevée pour une population qui aura le rôle de protéger la société contre les méfaits du tabac. Cependant une attention particulière doit aussi être accordée à la consommation grandissante de la chicha dans notre société d'où la nécessité d'insister sur l'information (méfaits $\mathrm{du}$ tabac) et l'introduction de l'enseignement de la pathologie du tabac dans le programme des études médicales.
7. Ministère de la Sante Publique du Niger. Projet de programme national de lutte contre le tabagisme 2009 -2014, Janvier 2009, p1-21.

8. SOLTANI MS, BCHIR A. Comportement tabagique et attitudes des étudiants en médecine à Monastir en regard du tabac Revue des maladies respiratoires 2000 ; 17 :77- 82.

9. Campaign for Tobacco-Free kids. Le tabac : un obstacle au développement durable. 2015, p2-5.

10. THIAM N, MBENGUE M. Habitudes tabagiques des étudiants de médecine de l'école saint-Christopher Iba Mar Diop. Revue des maladies respiratoires 2015; 32:137.

11. KOUARA S. Tabagisme chez les étudiants de médecine de Fès [Thèse doctorat]. Fès : Université SIDI MOHAMMED BEN ABDELLAH ; 2012.

12. YASMINA Z. Connaissances, attitudes et pratiques vis-à-vis du tabagisme chez les étudiants de la faculté de Médecine de Marrakech [Thèse doctorat]. Marrakech : Université CADI AYYAD; 2007. 